JSRM Code: 013010200002

\title{
The Adaptability of Somatic Stem Cells: A Review
}

\author{
Tweedell KS ${ }^{1}$
}

Cell and tissue specific somatic stem cells develop as dynamic populations of precursor cells to discrete tissue and organ differentiation during embryonic and fetal stages and their potential evolves with development. Some of their progeny are sequestered into separate cell niches of tissues as adult somatic stem cells at various times during organ development and differentiation These are diverse cell populations of stem and progenitor cells that respond to homeostatic needs for cell and tissue maintenance and the cycling of differentiated cells for physiological/ endocrinological changes. Nominally, multipotent stem cells in one or more niches follow specific lineages of differentiation that can be followed by diverse markers of differentiation. The activation of precursors appears to be stochastic and results in a population of heterogeneous progenitor cells. When variations in the functional need of the tissue or organ occurs, the progenitor cells exhibit flexibility in their differentiation capacity. Regulation of the progenitors is the result of signals from the stem cell niche that can cause adaptive changes in the behavior or function of the stem -progenitor cell lineage. A possible mechanism may be alteration in the differentiation capacity of the resident or introduced cells. Certain quiescent stem cells also serve as a potential cell reservoir for trauma induced cell regeneration through adaptive changes in differentiation of stem cells, progenitor cells and differentiated cells. If the stem-progenitor cell population is normally depleted or destroyed by trauma, differentiated cells from the niche microenvironment can restore the specific stem potency which suggests the process of dedifferentiation.

Key Words: Somatic stem cell; Adaptation; Dedifferentiation; Plasticity; Niche

Adult somatic stem cells (SSCs) are self-renewing groups of cells in tissues and organs that can produce specific lineages of precursor cells leading to differentiated cell progeny. They are retained from organogenesis throughout life for cell maintenance, repair and regeneration $^{[1]}$. During differentiation SSCs are established in unique cell/ECM niches. Niches are tissue specific sites in vivo consisting of differentiated cells that modulate stem cells. The niche histological composition varies extensively in different tissues but often includes stromal cells, extracellular matrix, blood vessels, neurons and tissue related precursor differentiated cells ${ }^{[2]}$. The stem cell population is often a mixture of quiescent stem cells (or active stem cells) and progenitor cells in various levels of differentiation. The surrounding niche cells regulate stem and progenitor cells and serve both as a specific topographical and functional site ${ }^{[3,4,5]}$. SSCs are often multipotent and their lineage leads to uni-potent progenitors for terminal differentiated cells. Activated stem cells divide symmetrically to produce identical cells for self-renewal. Alternatively, an asymmetric cell division produces a reserve stem cell and a cytoplasmic partitioned progenitor cell committed to a specific pathway. Where there is a cyclic demand for somatic cell renewal, short lived, intermediate transit amplifying (TA) cells proliferate extensively before differentiation into adult cells ${ }^{[4,5]}$. The resident population of diverse progenitors is possibly recruited and selected for their developmental potential to meet a specific function. The niche modulates stem cell function needed to maintain physiological needs for homeostasis and organismic variations in growth, maturation, reproduction and senescence that can alter stem/progenitor behavior ${ }^{[6]}$. Stem cells and their progeny within the niche may also be transient rather than fixed and adaptable to unusual conditions during tissue homeostasis or trauma that affects or depletes the cell population ${ }^{[7]}$. Studies on quiescent stem cells indicate they are maintained by epigenetic, transcriptional and post-transcriptional controls ${ }^{[8]}$. Self-renewing stem cells are maintained by niche derived signals such as Wnt found in multiple mammalian tissues ${ }^{[9]}$. While developmental determination of the stem cells from the quiescent state to active renewing stem and progenitor cells follows a directional lineage, there are indications that the identity of the stem cell states is fluid and exhibits plasticity ${ }^{[10]}$. The co-existence of quiescent and active stem cells has been described ${ }^{[11]}$ and the interconversion of quiescent and active cells is bidirectional ${ }^{[12]}$. The concept of a stem cell as a discrete entity is evolving into that of a biological function with a degree of plasticity ${ }^{[13]}$. The functionality of stem cells has been broadened to include undifferentiated cells, facultative cells and differentiated cells ${ }^{[14]}$. A key feature of these changes in cell fate is differentiation. It is possible that the degree of differentiation can be manipulated both within a specific lineage and between niches, during normal or abnormal needs for repair. Several SSC systems are examined here for the multiple controlling factors that enable the natural progression of differentiation in the stem cell lineage and for evidence that the determined cells are adaptable possibly by dedifferentiation.

\section{Cell Specific Stem Cells}

\section{Hematopoietic Stem Cells}

In the adult mammal a number of separate stem cells are found in the bone marrow. A well characterized and utilized stem cell is that responsible for hematopoiesis, the hematopoietic stem cell (HSC). 
HSCs are found in the endosteal and perivascular regions of the bone marrow of the adult mammal and are precursors to blood cell components consisting of the lymphoid progenitors of the immune system and myeloid precursors to the multiple blood cell phenotypes. The process of hematopoiesis has its roots in embryonic and fetal stages, first occurring in the yolk sac, an area of the aorta-gonad mesonephros (AGM) then the placenta and next the fetal liver. Several signaling pathways occur including, Notch 1, regulated by a transcription of Runx1, and the CDX-HOX pathway. The interpretation of HSCs colonization is in a state of flux and evidence has implicated the fetal liver as their origin. One view has HSCs of the liver entering the adult bone marrow, a second states that they are seeded in both sites at the same time but direct tracking has not been demonstrated ${ }^{[15]}$. The fetal liver is the first tissue from which HSCs have been purified. In the fetal/adult bone marrow, the HSC niche is associated with trabecular bone closely linked to adherent osteoblasts. The niche is viewed as a mixture of HSCs, progenitor cells and stromal cells embedded in a well vascularized extracellular matrix (ECM). Adhesion to the osteoblasts is by a nuclear-cadherin/betacatenin complex.

Alternate HSC niches in the bone marrow are in perivascular sites proximal to the endothelium ${ }^{[6]}$. The $\mathrm{Wnt} /$ beta catenin and Notch-Delta pathways drive the adult HSC lineages. There are many molecular similarities between the fetal and adult HSC stem cells that were analyzed by DNA analysis using bioinformatics and hybridization techniques that outlines the complete molecular phenotype of the $\mathrm{HSC}^{[16]}$

Many primitive HSCs are quiescent or reserved, used for homeostasis or in response to injury while others are active cycling stem cells that replenish the rapid turnover of blood cells. The migration of HSCs from the fetal mouse niche in the liver into the adult bone marrow niche results in dramatic changes from an active proliferating state into a quiescent mode. It has been postulated that separate quiescent and active populations of HSCs coexist in the bone marrow ${ }^{[15]}$ and they are regulated as they migrate into each of them ${ }^{[11]}$. Studies with the chemokine CXCL12 (a protein that regulates the immune response and HSC migration) indicate that HSCs and progenitors are located in perivascular niches while lymphoid progenitors exist in endosteal niches ${ }^{[17]}$. A specific HSC marker has been found in mice, ESAM (endothelial cell selective adhesion molecule) that is retained for life. It is also expressed in human CD34+ cells recovered from human cord blood ${ }^{[18]}$. Supporting cells in the bone marrow niche, osteoblasts and osteoclasts, mesenchymal progenitors and vascular (reticular) cells, regulate the homeostasis and activation of these stem cells $^{[15]}$. HSCs are identified initially by the detection of cell surface markers that express antigenic clusters of differentiation (CDs). Those found in animals often differ from CDs in the human although there are some that are conserved with murine animals. In addition, there is often overlap with those found in other tissues. As self-renewal of stem cells ceases, progenitors form, differentiate and the CDs expressed also changes. In the human, the CD34+is the primary clinical marker for HSC/progenitors. It is often expressed with the lineage antigen (Lin-) in combination with CD38+, CD45, CD90, CD133 along with Tie (angiopoietin-receptor) and c-kit (tyrosine kinase KIT) ${ }^{[19]}$.

A compendium of phenotypes in the HSC lineage through successive levels of differentiation to the ten terminal differentiated blood cells has been described for both murine animals and the human. The catalog of CDs differs in either species but the individual phenotypes between the replicating stem cells and the first multipotent progenitor is remarkably similar within each species. The self renewal of mouse HSCs expresses a phenotype of Lin-, c-kit, Flk2-. CD34 and Slamf1(receptors for HSC stem and early progenitors ). The human cell array shows CD38-, CD90+ and CD45RA- plus CD34+ and Lin-. Two surface markers, CD34 and Lin- are conserved in the two species and their presence extends from the activated stem and progenitor lineage to the final restricted progenitors for terminal differentiated cells ${ }^{[20]}$. Normal HSCs has been found in various niches from bone to cord blood. CD44 is involved in adhesion of cells to the ECM by hyaluron and is a receptor for the cytokine osteopontin (Opn) in the bone marrow. CD 90 is related to stem cell quiescence and CD 123 serves stem cell differentiation ${ }^{[20]}$ (Table 1).

Another parameter of $\mathrm{HC}$ stem cell specificity are signaling molecular pathways whose function is to enable HSC renewal as well as promoting differentiation lineages. The canonical Wnt pathway promotes the emergence of HSCs from the mesodermal endothelium of the dorsal aorta during early development. Niche cells communicate with their stem cell components through individual clusters of signaling molecular pathways. Another link (Tie receptor/angiopoietin 1) regulates HSC adhesion and promotes quiescence while $(\mathrm{Opn})$ from osteoblast cells down regulates HSC proliferation. Niche osteoblasts also express an $\mathrm{N}$-cadherin/ $\beta$-catenin complex thought to mediate attachment of HSCs in their niche. A proposed Wnt signal-nuclear $\beta$-catenin sequence regulates HSC proliferation while c-Myc interaction with Notch signals appears to regulate HSC differentiation ${ }^{[6]}$. In the adult, quiescent stem cells in the endosteal zone are maintained by inhibitory signals such as BMP, OPN and sFRP1. Active stem cells in the central zone are stimulated by Wnt, fibroblast growth factor (FGF) and SDF1 (stromal derived epidermal factor) pathways from endothelial cells, megakaryocytes and reticular cells ${ }^{[11]}$.

The local niche environment may accomplish stem cell maintenance of HSC's with cell-cell contact or at a distance ${ }^{[19]}$. The effect of niche influence on resident HSC stem cells is extensive. Murine granulocyte macrophage cytokines can instruct the hematopoietic lineage ${ }^{[21]}$. The stem cell factor (SCF), is produced by perivascular mesenchymal cells and endothelial cells that maintain HSC's in both embryonic and adult tissues. This is a key perivascular niche that provides multiple cell types during homeostasis ${ }^{[22]}$. When the stem cell population is depleted, newly introduced stem cells behave as the original cells ${ }^{[19]}$, an attribute found in developing organ primordia and shared by the blastemal cells in the regenerating vertebrate limb ${ }^{[23]}$

It had been generally held that a common pluripotent primitive stem cell produces two lines of multipotent stem cells, progenitors to the lymphoid immune system cells and the other forms myeloid stem cells, with eight precursors to a multiple blood cell lineage ${ }^{[15]}$. The original concept predicted a long term lineage sequence of oligopotent progenitors into unipotent progenitors. The classical view that multipotent HSCs produce multipotent progenitors for the separate common myeloid progenitor (CMP) and lymphoid lineages has been modified for both the murine fetal liver and adult bone marrow ${ }^{[24]}$.

The niche of the fetal mouse liver contains HSC stem cells and associated pericytes that express Nestin and a neural/glial antigen 2 (NGA2) when both cells are in a high proliferation state ${ }^{[25]}$. With continued development, the cells move into a HSC niche of the adult bone marrow where the HSCs and pericytes evolve into a quiescent state. Concomitantly, two studies have provided evidence that multipotent common myeloid progenitors follow a different differentiation sequence in the fetal mouse niche compared to the adult niche. Single cell culture assays ${ }^{[26]}$ showed the presence of oligopotent cells in the fetal niche that do not exist in the definitive adult state. This view was substantiated in a second investigation ${ }^{\text {[27] }}$ with single-cell transcriptome analysis of 2700 myeloid progenitors in the mouse. The analysis failed to detect any type of CMP cells with multipotent progenitor ability in the adult. 
Table 1: Somatic stem cell constituent markers and pathways

SOMATIC STEM CELL CONSTITUENT MARKERS

\begin{tabular}{|c|c|c|c|c|c|c|}
\hline $\begin{array}{l}\text { Stem } \\
\text { Cell }\end{array}$ & $\begin{array}{l}\text { State: } \\
\text { STAGE }\end{array}$ & Quiescent & Active & $\begin{array}{c}\text { Progenitors - } \\
\text { Differentiation }\end{array}$ & Components & Func \\
\hline \multirow[t]{2}{*}{ HSC } & Develop. & $\mathrm{CHD}^{[39]}$ & $\begin{array}{l}\text { CXCL-12 } 2^{[15,17]} \\
\text { SCF }^{[22]} \\
\end{array}$ & & $\begin{array}{c}\text { Wnt,FGF,SDF-1 } \\
\text { Notch1, RunX, CDX-HOX, } \\
\text { PGE2 } 2^{[15]}\end{array}$ & Q A \\
\hline & Adult & $\begin{array}{c}\text { BMP,OPN, } \\
\text { FRP1 }^{[11]}\end{array}$ & $\begin{array}{l}\text { SCF, SCA1+, } \\
\text { CD34-,LIN,Flk2, } \\
\text { Slamf1-, ckit }{ }^{220]} \\
\text { H: Lin-,CD34, } \\
\text { CD45RA-,CD38- } \\
\text {,CD 90 }\end{array}$ & $\begin{array}{c}\text { Sca 1+, Flk2, c-kit, Lin -, Slamf1+, } \\
\text { CD27+, CD34++ }{ }^{[20]} \\
\text { IL7RA++[19] } \\
\text { ESAM }^{[18]} \\
\text { NG2, Nestin }{ }^{[25]} \\
\text { H: Lin, c-kit, IL3RA, Cd10+.CD38, } \\
\text { CD45RA, CD90, CD334+, } \\
\text { CD123,Tie. }{ }^{[19,20]}\end{array}$ & $\begin{array}{l}\text { OPN, BMP,FRP } \\
\text { Wnt/N/beta catenin,c-myc } \\
\text { Notch }^{[6]} \\
\text { Wnt, FGF, SDF-1 } \\
\text { Wnt/beta catenin ,Notch, } \\
\text { Delta } 111^{[11]}\end{array}$ & $\begin{array}{l}\text { A } \\
\text { Df }\end{array}$ \\
\hline \multirow[t]{2}{*}{$\begin{array}{l}\mathrm{NSC} / \\
\mathrm{NCC}\end{array}$} & Develop. & & & $\begin{array}{l}\text { Sox2, Pax6,Nestin, GFAP GLT-1,CBP, } \\
\text { BLBP, Vimentin }{ }^{[29,30]}\end{array}$ & $\begin{array}{l}\text { Wnt/catenin. SHH, BMP, } \\
\text { RA,FGF }\end{array}$ & Df \\
\hline & Adult & $\mathrm{CHD} 7^{[39]}$ & & $\begin{array}{c}\text { GLAST,CBP,GFAP,BLBP,GLT1,TGF, } \\
\text { FGF, RA,TN-.C,RC- } \\
\text { 1,2,Nestin,Vimentin.,GS, } \\
\text { Pdgf }{ }^{[30,34,35,40]} \\
\end{array}$ & $\begin{array}{c}\text { Notch/delta ll1,Wnt, Jag.1, } \\
\text { Ephrin B }{ }^{[37,38]} \\
\text { Wnt/catenin,SHH,TGAF,T } \\
\text { FG,PDGF,EGFR }{ }^{[40,42,45]}\end{array}$ & Q Df \\
\hline \multirow[t]{2}{*}{ PrSC } & Develop. & & $\operatorname{Lgr}^{[49,58]}$ & Lgr4, Lin-, Scz1+, CD49 $9^{[49,58]}$ & & A \\
\hline & Adult & & & $\begin{array}{c}\text { ckit, CD44, CD133, CD117 } 7^{[51]} \\
\text { CD 138, CD49f, K5, K8, K14,K18, } \\
\text { PSA }{ }^{[56]} \\
\text { H CD133, TROP 2,CD44, CD49f }{ }^{[53,54]}\end{array}$ & $\begin{array}{l}\text { Alpha } 6 \text { Integrin }{ }^{[50]} \\
\text { Wnt, Notch/Delta, } \\
\text { Jagged1, SHH }{ }^{[57,58]} \\
\text { H: alpha/beta integrin }{ }^{[53]}\end{array}$ & Df \\
\hline \multirow[t]{5}{*}{ MaSC } & Develop. & & & K14,K19 ${ }^{[61,62]}$ & Wnt & $\mathrm{Df}$ \\
\hline & Neonatal & & & K5,K8,K14+,K19+,EGRF[62,63,65] & & Df \\
\hline & Adult & $\operatorname{Laminin}^{[61]}$ & & $\begin{array}{c}\mathrm{K} 5, \mathrm{~K} 8, \mathrm{~K} 14+, \mathrm{K} 19+, \text { lin-, CD24, } \\
\mathrm{CD} 29+ \\
\mathrm{CD} 29-, \mathrm{CD} 49+_{+}{ }^{[61,62,65]}\end{array}$ & $\begin{array}{l}\text { Notch/Jag. 1, Wnt, Integrins, } \\
\text { EGFR, E,P cadherins } \text { s }^{[63,67]}\end{array}$ & Q \\
\hline & Pregnancy & & & $\mathrm{K} 8, \mathrm{~K} 14, \mathrm{~K} 18, \mathrm{CD} 24, \mathrm{CD} 29^{[65]}$ & $\begin{array}{l}\text { Wnt, Notch/Delta, SHH, } \\
\text { HOX,FGF,TGF,p } \\
\text { Cadherin }^{[67]}\end{array}$ & Df \\
\hline & Lactation & & & $\mathrm{K} 14, \mathrm{~K} 18, \mathrm{p} 63, \beta$-casein ${ }^{[64]}$ & & Df \\
\hline \multirow[t]{2}{*}{ ISC } & Develop. & LRC $^{[74]}$ & & Lgr5 & Wnt,SHH,Hox,FGF,TGF ${ }^{[69]}$ & Df \\
\hline & Adult & \begin{tabular}{c|}
$\mathrm{Bmi1}$ \\
$\mathrm{H} 2 \mathrm{~B}^{[78,79,83]}$ \\
$\operatorname{Lgr}^{[12,73]}$
\end{tabular} & $\begin{array}{l}\text { Lgr5 }^{[12,74,77,83]} \\
\text { Bmi1, Hopx, Sox } 9 \\
\text { mTert,Lrig } 1^{[79,82]}\end{array}$ & Lgr5, Delta 111, Bmi1 [82] & $\begin{array}{c}\text { Notch/Delta } 1^{[80,82]} \\
\text { Wnt-,Wnt+, Wnt+/catenin, } \\
\text { EGFR }^{[83]}\end{array}$ & $\begin{array}{l}\text { Q,A } \\
\text { Df }\end{array}$ \\
\hline
\end{tabular}

H: human, Q: quiescent, A: Active, Df. Differentiation. [xx]: Reference 
Rather, the HSC compartment produces individual unipotent progenitors for all myeloid, erythroid and megakaryocyte progeny. Intermediate oligopotent precursors are replaced by individual unipotent progenitors for lymphoid progenitors, $\mathrm{T}$ and $\mathrm{B}$ lymphocytes and natural killer cells. HSCs evolve with a sequence of developmental sites as they age. While stem and progenitor cells follow a specific lineage, their function may vary as dictated by cell regulatory components in separate osteoblast and vascular niches. HSCs are viewed as groups of related cells, with varying potential, controlled by transcription factors and input from separate, adjoining cellular niches. The active population of HSCs maintains the cycling blood cells while the quiescent population responds to acute cell $\operatorname{loss}^{[18]}$

\section{Neural Stem Cells}

In mammals, morphogenetic and cellular changes in the embryonic neural tube form the primitive forebrain (telencephalon) mid-brain and hind-brain. Neurogenesis is initiated in the ventricular zone of the brain wall when concurrent changes in cell shape and migration are associated with the formation of embryonic neuroepithelial (NE) cells, the source of primary neural stem cells in the developing brain that are multipotent progenitors to neurons and glial cells ${ }^{[28]}$. Early symmetrical divisions of the proliferating embryonic NE cells express specific neural cell markers, Sox2, Pax6 and the production of Nestin (an intermediate filament protein) and a glial fibrillary acetic protein (GFAP) marker ${ }^{[29]}$. Early on, NE cells along the subventricular zone (SVZ) produce progenitors to neurons followed by asymmetric divisions leading to more restricted progenitors of radial glial (RG) cells that multiply and eventually replace all NE cells ${ }^{[30]}$. The RG cells also exhibit characteristics of astroglial cells, a glutamate transporter (GLT1), Ca+ binding protein (CBP), GFAP, vimentin and a brain lipid binding protein (BLPP) ${ }^{[30]}$. Cre recombinase (Cre-lox) mapping demonstrated that these NE cells are the source of neural stem cells (NSCs) since they can form both neurons and radial glial cells (RF) in the embryo ${ }^{[31]}$.In the developing brain, RG cells form astrocytes but also form neurons. From these observations, a unified cell continuum of stem cell formation, from the embryo to the adult, was proposed resulting in a neuroepithelial-radial glia-astrocyte lineage ${ }^{[32]}$. Embryonic neurogenesis in the cortex proceeds by asymmetric division that produces an RG cell and a neuron progenitor or indirectly through one or two divisions of intermediate progenitor cells for neurons in the ventricular zone (VZ). Heterogeneous neurons which differ according to their locality or their developmental stage are controlled by activation of an extensive range of transcription factors. Furthermore, the RG cells produce separate intermediate progenitor cells that generate either neurons or astrocytes during embryonic development and in the adult ${ }^{[33,34]}$.

In the neonatal stage, RG cells continue to produce neurons and oligodendrocytes via intermediate progenitor cells. A sub-population of RG cells, known as B cells, convert into astrocytes in the (SVZ). These are the source of NSCs in the adult and generate neural intermediate progenitor cells that culminate in neuroblasts (precursor neurons). The RG cells express astroglial markers: GLASt, BLBP, Tenascin C (TN-C),GFAP, Nestin, Vimentin, RC1,2. Other astrocytelike neural stem cells, originally derived from embryonic RG cells also have glial cell properties ${ }^{[34]}$. These adult astrocyte-like NSCs appear to be pre-determined for a specific neuronal or glial fate ${ }^{[35]}$. In the post- natal rodent brain the radial glia-astrocyte stem cell sequence yields neurons, ependymal cells and astrocytes in two niches of the adult brain One region is located in the brain wall adjacent to the lateral ventricles of the forebrain, the sub-ventricular zone (V-SVZ), that ultimately generates olfactory bulb interneurons. A second is found in the subgranular zone (SGZ) the dentate gyrus of the brain hippocampus ${ }^{[29,34]}$. Neural intermediate progenitor cells generate diverse neurons and intermediate glial progenitor cells form heterogeneous oligodendrocytes ${ }^{[34,35]}$. Other evidence has indicated that similar niches exist in circum-ventricular organs (CVOs) along the ventricular midline and in novel niches along the 3rd and 4th ventricles in the adult ${ }^{[36]}$. See Table I and Abbreviation List.

Components of the neural stem cell niche promote quiescence and insure stem cell identity. Neural stem cells in the SVZ niche depend upon direct cell to cell contact with endothelial cells of the vascular niche. Two proteins, endothelial ephrin B2 and Jagged 1 prevent the production of mitogens that inhibit differentiation ${ }^{[37]}$. Adult mouse quiescent NSCs in the subventricular zone are also reinforced by the induction of the Notch ligand Delta-like 1 (Delta 1l 1) signaling pathway in activated NSCs, presumably by feedback on the reserve stem cells ${ }^{[38]}$. In the hippocampus a chromatin remodeling factor, chromo-domain-helicase-DNA binding protein 7 (CHD7), represses the transcription of several positive regulators of cell cycle induction $^{[39]}$. Stem cell activation leads to their repression allowing stem cell renewal and neurogenesis of adult neurons and glial cells (astrocytes and oligodendrocytes) ${ }^{[40]}$. The diversity of the neural stem cell populations found in adult neural niches reflects the plasticity of the NSCs. They consist of sequential cell stages, Type B1 astrocytes (radial astrocytes), that develop Type $\mathrm{C}$ transit amplifying cells (intermediate progenitors) which divide to form Type A granule cells (neuroblasts), precursors of differentiated neurons ${ }^{[29,40-43]}$. There are three different compartments (domains) of the adult V-SVZ niche which allow for regulation of stem cell function during homeostasis, cell regeneration and aging ${ }^{[42]}$. The ventricular chamber is lined with multi-ciliated ependymal cells (B1) in the first domain (DI). Moving inward into the ventricular wall, domain II (DII), B1 astrocyte cells form transit amplifying "C" cells that divide to form A cells (neuroblasts). In the third innermost zone (DIII), B1 cells are in communication with blood vessels via extended cell processes ${ }^{[40,43]}$. A variety of growth factors, ligands and signaling pathways appear to regulate $\mathrm{B} 1$ cells and progenitor cells behavior in all three domains ${ }^{[42]}$. Many soluble factors (TGFs, IGFs, PDGFs, BMPs, Wnt, SHH) and retinoic acid are found in the cerebral spinal fluid (CSF) that can affect cell proliferation, neurogenesis and oligodendrogenesis ${ }^{[40,42,43]}$. The ependymal cells secret noggin which enhances progenitor cells and neuroblast formation ${ }^{[42]}$. Other factors are released from blood and endothelial cells. Notch signaling is viewed as bidirectional between niche cells, stem cells and their progeny during homeostasis and regeneration ${ }^{[40]}$. For example, neurotransmitters from serotoninergic axons appear to regulate interactions between B1 cells, progenitor cells and newly formed neuroblasts ${ }^{[43]}$.

In the SGV of the brain hippocampus a different niche compartment has been described with multiple pathways for radial cell development ${ }^{[42]}$. Three similar domains of radial astrocytes (RA) are located within the dentate gyrus, DI, a subgranular zone adjacent to blood vessels, DII, a granular zone where RAs interact with their progeny and DIII, an inner molecular zone that exposes RA cell extensions to neuronal networks. Since the gyrus lacks a ventricle, Domain I in the gyrus is the Hilus, a deep region of intermediate progenitor cells (IPC) and blood vessels. Here, factors from blood vessels, endothelial cells and RA cells stimulate RA type 1 cells to form intermediate progenitor cells (IPCs) that migrate into the upper granular zone. Domain II consists of the RA cell bodies that are anchored with a primary cilium in the hilus along with a mixed lineage of IPCs (type 1 and 2) and granule (type 3) cells. When mature, granule cells become anchored by an axon into the Hilus and apically send dendrites into the inner molecular layer of domain III. 
The RA cell bodies also extend projections into the molecular layer forming dense branches of mossy fiber axons. In domain II there is cell-cell interaction between NSCs, their progeny and granules cells and their behavior is regulated by various neuro-transmitters such as (GABA). Domain III is an inner molecular layer of other glial cells, axons and synaptic terminals. The two neurogenic niches of neural stem cells harbor B1 astrocytes in the SVZ and radial astrocytes in the SGZ. The functions of quiescence, cell proliferation and progenitor differentiation appears to be regulated by specific cell to cell interaction with their progeny and soluble factors from blood vessels, the ventricular fluid and neuronal interchange that provide alternative avenues for niche regulation. The multiple signaling pathways remain to be identified in vivo and may cross regulate on the same cell ${ }^{[42]}$. These and other biophysical properties of the dynamic niches control neural stem cell function during homeostasis, and regeneration ${ }^{[40,43]}$ and neurogenesis in both SVZ and SGV declines with age ${ }^{[42]}$.

\section{Neural Crest Precursors}

Other neural stem cell derivatives originate from the neural crest cells (NCC), a cache of pluripotent migratory stem cells that arise from the lateral margins of the embryonic brain and spinal cord. They form the sensory and motor neurons, ganglia of the peripheral and enteric nervous system, glial cells, melanocytes, endocrine cells and diverse types of mesenchyme cells. Another small subset of stromal cells originating from NC cells express the protein Nestin and also contribute to the maintenance of HSCs ${ }^{[22]}$. Early experiments by Le Douarin et al. on chick-embryo/quail - adult chimeras indicated that NCC differentiate into a specific type of glial cell that is dependent upon the microenvironment where they develop ${ }^{[44]}$. Another quality of differentiated cells from the NCC lineage is their plasticity in the adult. The differentiation of glial cells and melanocytes can be reversed when the cells are returned to an intermediate precursor. A reciprocal conversion between glial cells and melanocytes occurs by dedifferentiation into a multipotent progenitor ${ }^{[44]}$. The most widely distributed glial cells are Schwann cells that surround axons with myelin throughout the body. In the adult, Schwann cells are also found to dedifferentiate into stem-like cells in response to injury, reverting to a precursor state that promotes new axon regeneration and re-myelination of injured neurons ${ }^{[45]}$. Adult Schwann cells may act upon perivascular smooth muscle and invade the bone marrow hematopoietic stem cell niche, secreting a transforming growth factorbeta that keeps the stem cells quiescent ${ }^{[45]}$. The wide spread glial plasticity between cell and tissue interactions of NCCs and non-NC derivatives of perivascular muscle, bone marrow and mesentery during development, injury and malignancy implicates NCC stem cell-like cells in diseases and neoplasia. The behavior and defects in NCCs or their interactions with its environment may help in the etiology of several diseases ${ }^{[46]}$. For example, Schwann cells will dedifferentiate in diseases such as leprosy, caused by Myobacteriium leprae. This disease causes Schwann cells to dedifferentiate, lose their glial identity and transform into highly migratory "stem cell-like cells". The Schwann cell precursors can then differentiate into new cells in vivo.

\section{Prostate gland stem cells}

The primordium of the prostate gland arises from an interaction of embryonic endodermal buds that develop multiple solid rod branches within a mesenchyme capsular cell mass. Androgen production ultimately controls epithelial morphogenesis of the solid tubular rods and buds which leads to duct formation within the buds followed by epithelial cell differentiation into basal cells and secretory luminal cells that face the duct lumen. Rare neuroendocrine cells in the layer, extend from the basement membrane between the luminal cells.
An early prediction of prostate stem cells (PrSC) was obtained when dissociated prostate epithelial cells and embryonic urogenital sinus mesenchyme fragments were derived from murine embryonic or adult donors and implanted into the kidney subcapsular space or implanted intra-muscularly in adult mice. Prostatic branching tubules were regenerated in the implants along with prostate cell markers ${ }^{[48]}$. Later, prostate tissue cells in a basal phenotype, identified as stem cells by the antigenic profile Lin- (mixed lineage marker), SCA1+(stem cell protein antigen) and CD 49f were isolated by FACS and cultivated in vitro. When combined with inductive stroma and implanted into the sub-capsule of the kidney or into the skin of immune-deficient mice, they regenerated tubules with an outer basal layer, an inner luminal layer and neuro-endocrine cells ${ }^{[49]}$.

Other isolations with in vitro cultivation of prostate cells utilized a variety of generic stem cell markers, and putative specific prostate stem cell combinations. Purified cells from the proximal duct with SCA-1 (stem cell protein antigen) that often co-expressed alpha 6 integrin were most effective in restoring the prostate ${ }^{[50]}$. Single cell isolates from the proximal urethra also expressed stem cell receptors (Lin(-) SCA-1+, c-kit, CDs 44, 133). They and a PC marker CD 117 , can generate secretion -producing prostate tissue in vivo ${ }^{[51]}$. These studies of prostate basal cell and luminal cells resulted in regeneration of secretory prostate cells when implanted in vivo. The prostate stem cell niches were located in the basal cell layer, proximal to the urethra ${ }^{[52]}$ and in the tip of proximal prostatic ducts ${ }^{[50,51]}$. In the human prostate, a subset of basal cells expressing alpha2/beta 1 integrin, a prostate cell surface marker and CD133 would produce prostate-like acini when transplanted into immune-compromised mice ${ }^{[53]}$. Similarly, a subpopulation of human prostate basal epithelial cells, expressing (Trop2), CD44 and CD49f, formed self-renewing prostaspheres in vitro that were implanted along with rat urogenital sinus mesenchyme into immunocompromised mice. Tubular structures formed in the implants that differentiated into discrete basal and luminal cells in vivo $^{[54]}$.

Based on the murine and human data Goldstein proposed a prostate epithelial hierarchy where basal stem cells produce a multipotent progenitor that generates all three types of epithelial cells ${ }^{[55]}$. During post-natal development, lineage tracing experiments on the prostate epithelium of mice verified that basal multipotent stem cells (intermediate cells) do differentiate into three main lineages. Basal cells expressed keratin (K), K5 and K14 along with a common transcription factor, p63, whereas luminal or secretory cells expressed $\mathrm{K} 8$ and $\mathrm{K} 18$ plus an androgen receptor, a prostate-specific antigen and prostate acid phosphatase and thirdly, neuroendocrine cells produced synaptophysin, chromogranin A and neuropeptides. Both unipotent basal progenitors and unipotent luminal progenitors were also present ${ }^{[56]}$. During homeostasis in the adult there is a shift from multipotent progenitors to separate unipotent basal and luminal cell progenitors as stem cell sources. Induction of prostate cell regeneration also results from activation of unipotent stem cell progenitors $^{[56]}$.

When prostaspheres and an in vivo prostate reconstitution assay were combined, the Wnt pathway induces Sc-1+, CD49f + basal/ stem cell proliferation. Up regulation of target genes led to an increase in $\mathrm{K} 5+$, $\mathrm{K} 8+$, p63+ prostate progenitor cells. Induction of Notch signaling in prostate progenitors inhibits their proliferation and normal morphogenesis. The opposing roles of Wnt/ Notch signals is attributed to modulation of Notch by Wnt signaling ${ }^{[57]}$. Early development of the prostate gland (which is post-natal in rodents) in Lin/Sca1+/CD49f+ basal stem cells, is associated with the expression of of Lgr4 (a G-protein coupled receptor) that occurs prior to any 
lineage differentiation in the neonatal gland. Yet, continued morphogenesis and differentiation is regulated by LGr4 expression. Notch and sonic hedgehog $(\mathrm{SHH})$ pathways are key targets in prostate stem cells and progenitors, both promoting morphogenesis and early differentiation. Lgr4 expression modulates both cell proliferation and differentiation through $\mathrm{Wnt} /$ Bcatenin signaling. Induction of Wnt signaling in basal stem cells down regulates Notch and blocks proliferation and morphogenesis of acini but induction of Notch in progenitors inhibits their proliferation. The inactivation or ablation of Lgdr4 down loads both Notch 1 and SHH expression and also blocks both morphogenesis and differentiation. It appears that Wnt modulation of Notch regulates proliferation and maintenance of epithelial progenitors ${ }^{[58]}$. Based on the multiple stem cell-progenitor involvement in prostate homeostasis and regeneration, it has been hypothesized that any progenitor cell can produce cells leading to differentiated secretory luminal cells. Moreover, the oncogenic transformation of these precursor cells is the basis for the genotypic variation and degree of malignancy found in prostatic cancer ${ }^{[59]}$.

\section{Mammary Gland Stem Cells}

Development of the mammary gland has primordial origins analogous to that of the prostate gland. Ectodermal cells forms primary solid cellular buds that subsequently branch within external mesenchymal tissue. At the fetal-neonatal stage two hollow chambers exist, a multibranched terminal lobular unit and a basal terminal end chamber. The mammary gland epithelium becomes organized into a layer of luminal-alveolar secretory cells and a layer of basal myoepithelial (MEP) cells that interact with the ECM. The basal epithelial cells are controlled by the beta1-integrin gene and these cells regulate the growth and differentiation of the luminal secretory cells ${ }^{[60]}$. Evidence of a multipotent mammary stem cell, capable of producing the entire mammary branching network was provided by single cell isolations from 8 week old mice. A subpopulation of cells isolated by FACS, were identified by Lin, CD29hi, CD24+ markers. A majority of these cells expressed the myoepithelial cytokeratin 14 (K14) marker. Double sorted Lin-, CD29hi, CD24+ cells were recovered, marked with a LacZ transgene, cultivated into buds in vitro and then transplanted into mammary gland fat pads where 6/102 recipients developed ductal structures of both luminal and myoepithelial cells. Further differentiation of lobulo-alveolar components occurred when transplants were made into adults during pregnancy and parturition. A separate cell population expressed the luminal cell marker, K18 and additional analysis indicated their cell progeny had a luminal cell fate when exposed to lactogenic conditions. The single cell isolates of self-replicating, multipotent stem cells, were defined as mammary gland stem cells (MaSCs) ${ }^{[61]}$.

The human breast stem cell hierarchy changes as gland development proceeds and increases in complexity from the fetal -neonatal stage, into the adult gland and during pregnancy. These cells are identified by different cell surface markers, CD24, CD29, CD44, CD49f and EpCAM. The developmental sequence can be followed by changes in cytokeratin staining (K). In the early fetus, luminal and basal cell lineages are established that are double negative for K14 and K19 markers. At the neonatal stage, multipotent progenitors, K14+, K19+ are produced by ductal/lobular cell and myoepithelial cells. In the adult, multipotent progenitor cells are recognized by keratin markers for two types of ductal lobular cells and a basal/myoepithelial cells ${ }^{[62]}$.

The basal epithelium appears to be the source of MaSCs, progenitor cells and differentiated basal cells. Isolation of basal cells from the mammary gland using Lin-, CD24+. CD29hi from virgin mice identified multipotent MaSCs with a more specific cell marker, a transmembrane endothelial protein $\mathrm{C}$ receptor (EPCR) for a Wnt target in the mammary gland from "Matrigel" cell cultures. Upon transplantation, these cells regenerated mammary gland components and also acted a multipotent stem cells in physiological lineage tracing with a knock-in allele of the EPCR receptor ${ }^{[63]}$. Another parameter of MaSC adaptation appears during mammary gland lactation. Based on the recovery of breast stem cells from human breast milk and analysis by extensive in vitro testing looking for the expression of human embryonic stem cell (hESCs) transcription factors. A proposed lineage from a common multipotent stem cell, $\mathrm{CD} 49 \mathrm{f}$, is the source for generating separate branches of luminal or myoepithelial progenitors. The luminal progenitor develops into ductal cells (K 19) or an alveolar luminal cell progenitor (K18). During lactation, the latter differentiates into a novel K18/B- casein, an a-lactalbumin secretory cell. Myoepithelial cells (K14) originate from separate progenitors ${ }^{[64]}$. Progenitors of the mammary gland are frequently modified due to the cyclic nature of the mammary gland during each menstrual (estrus) cycle. Evidence from genetic lineage studies indicates that the stem cell identity varies from the fetal to neo-natal, during homeostasis and pregnancy ${ }^{[64]}$. As a result, there is a special relationship between the adult basal stem cells and their micro-environmental niche that separates them from neonatal stem cells.

A group of lineage tracing experiments and clonal analysis of the mouse mammary gland were performed in situ, under physiological conditions, on embryonic, adult and pregnancy phases of the mammary glands. Using keratin gene derived K14, yellow fluorescent proteins (YFP+) cells it was confirmed that all mammary gland epithelial lineages were derived from multipotent K14 expressing progenitors which included myo-epithelial and luminal cells during embryonic development. At the beginning of puberty and during homeostasis, it was found that two types of lineage restricted cells differentiated into either myoepithelial or liminal cell lineages. In vivo experiments based on immune-staining indicated that cells during puberty and in adult virgin mice which expressed $\mathrm{K}$ 14 , were limited to myoepithelial cells. This continued into adult life and during multiple pregnancies. No K-14 expressing cells contributed to the luminal lineage during their expansion at puberty. In contrast, K-8/YFP+ expressing stem cells were limited to the luminal cell lineage during puberty, adult gland homeostasis and continued through 3 consecutive cycles of pregnancy and lactation. These separate, long lived, unipotent stem cells also demonstrated massive expansion during pregnancy ${ }^{[65]}$. New investigations on the in situ in changes of the mouse mammary gland utilized a multicolored Cre reporter gene and observations by 3D confocal imaging, combined with specific markers and immuno-staining in several transgenic mice that allowed in vivo identification of both stem cells and progenitors ${ }^{[6]}$. The contribution of progenitor cells at different stages during development and the adult was determined by producing transgenic strains specific to basal and luminal lineages, using green fluorescent protein (GFP) linked-to a reverse tetracycline transcriptional activator (tTA) and an internal ribosome entry site, (GFP-rTIR). The transcription factor Elf5, from a signature gene for mouse and human alveolar tissue differentiation, was employed. FACs analysis showed that the Elf5-GFP was confined to the luminal layer but not the basal/MaSC cell population in pubertal and adult mammary glands. Tracing luminal progenitor cells in vivo indicated they induced alveologenesis which expanded during puberty. The basal cell population of MaSCs and myoepithelial followed with keratin K5 and FACS analysis using GFP showed they were confined to myoepithelial cells. Bipotent K5 expressing cells cells were tracked and found during morphogenesis, puberty and homeostasis in the adult. The fate of restricted luminal progenitors utilized the same 
triple-transgenic mice combined with floxed multicolored (confetti) reporter alleles. Elf5-labeled cells contribute to alveolar cells in pregnancy and about $60 \%$ of them express 2-4 fluorescent proteins. The data promoted a common basal cell precursor, a bipotent mammary gland stem cell, which yielded both myoepithelial and luminal cells. These bipotent stem cells were active during morphogenesis, homeostasis in the adult mouse, and physiological changes during pregnancy and lactation. The lineage of the unique progenitor cells supports a model that states cell replenishment is initiated by MaSCs and continued by long lived unipotent progenitors $^{[66]}$.

Several signaling pathways control mammary stem cell development, particularly progenitor cells that maintain homeostasis. Niche-derived mammary gland proteins and ECM molecules were found to specify bipotent human progenitor cells ${ }^{[67]}$. Cell cultures of human progenitor cells indicated the niche maintained quiescence, the progenitor state and directed differentiation toward myoepithelial and luminal stages. Progenitor cell fate occurred through integrin signaling along with Notch, EGFR and both E- and P-cadherins. It was concluded that the regulation does not dictate pre-determined programs but the niche imposes specific behavior upon them. In vivo analysis indicated Laminin 1 maintains quiescent cells. The Notch ligand, Jagged 1 imposed the $\mathrm{K} 14+/ \mathrm{K} 19$ progenitor phenotypes and with $\mathrm{P}$ - cadherin selected the K14+/K19+ phenotype in the MEP cells ${ }^{[67]}$. The mammary stem cell niche can also modulate the differentiation response of other unrelated stem cell hierarchies when they are transplanted into the mammary niche that further reflects their plasticity (see discussion) ${ }^{[68]}$. This niche regulatory function also parallels the regulatory role of the early regeneration blastema on introduced foreign primordia ${ }^{[23]}$ and the reactivation of ectopic cells in a vacant Drosophila niche ${ }^{[20]}$. Yet, the neoplastic conversion of mammary stem cells as potential cancer cell precursors provides a parallel to lineage studies on developing mammary cells. An antibody study on human breast cell origin found that normal epithelial K19+ luminal cells from the breast are localized in $>95 \%$ of human breast carcinomas $^{[62]}$.

\section{Intestinal Stem Cells}

The mucosal lining of the small intestine in the late embryo consists of a continuous epithelium, along the intestinal lumen, that develops multiple finger-like projections of villi separated by crypts between their bases. In the crypt, the epithelium forms crypt based columnar cells $(\mathrm{CBC})$ and by the late embryo/early post-natal period, the crypts have stem cells and progenitor cells along with Paneth cells at the crypt bottom. Gut development forms by an interaction of the Wnt canonical signaling pathway and the TGF- $\beta$ superfamily pathway along with FGF, hedgehog ( $\mathrm{SSH})$ \& Hox proteins that control cell division and fate ${ }^{[69]}$. Proliferating cells in the embryonic crypt of mice are polyclonal, i.e. biparental, but early in postnatal life, cloned pools of adult stem cells result from asymmetric cell divisions. In the human adult, the intestinal crypts may also contain multipotent, self renewing stem cells capable of replacing millions of cells every few days. Adult homeostasis was postulated as regulation of stem cells and their progeny by niche derived signals, signaling pathways, transcription and growth factors that direct their fate ${ }^{[6,70]}$. An early study by Cheng and Leblond ${ }^{[71]}$ showed that the CBC cells, dispersed between Paneth cells, were long lived, self-renewing multipotent intestinal stem cells (ISCs) that maintain homeostasis of the differentiated cell types found along the entire villus epithelium. These terminal cells are enterocytes, goblet cells, entero-endocrine cells, tuft cells and $\mathrm{M}$ cells ${ }^{[72]}$. The cyclic replacement of the differentiated cells along the villi begins by cell amplification of progenitor transit amplifying (TA) cells in the crypt niche. The TA cells cycle into differentiated lineages as they migrate up the villus to replenish the differentiated cells in the epithelium. The existing absorptive, secretory and hormonal cells are replaced every 4 to 5 days after they undergo apoptosis and escalate into the intestinal lumen. Paneth cells remain in the crypt base where they produce an anti-microbial substance, nurture the ISCs and are replaced by transient cell precursors ${ }^{[73]}$. The marker gene for CBC cells, Lgr5+ (Leucine $\mathrm{G}$ protein receptor) identified the $\mathrm{CBC}$ cells as the active intestinal stem cells (ISC) in the crypt ${ }^{[74]}$. Activation of a Lac Z reporter gene showed that all cell types of the villus epithelium appearing in the ribbon of cells produced from the Lgr5+ marked gene in the CBC cells, were invariably in contact with Paneth cells ${ }^{[75]}$.

Yet, other observations by Potten et al. ${ }^{[76]}$ on chimeric mouse embryos proposed a stem cell model where active ISCs were located in the number +4 cell position from the crypt base, on the villus above the Paneth cells. They were identified as DNA (thymidine) label retaining (LRCs) that cycle every 4 hours. It was postulated that the +4 cells are a subset of LRCs, quiescent reserve DNA label retaining cells (LRCs) which are transient precursors of Paneth cells. When the ISC pool is depleted the LRCs can dedifferentiate into Lrg5+ ICS cells ${ }^{[73]}$. The quiescent and active stem cell subpopulations have separate functions, Lgr5+ for the former and Lgr5- in the latter ${ }^{[12]}$. They occupy separate yet adjoining niche locations ${ }^{[77]}$. A definitive lineage tracing of the +4 stem cells employed a marker, Bmi1, a proto- oncogene, which identified reserve, slow cycling stem cells that differed from the active cells ${ }^{[78]}$. The two stem cell populations showed an interconversion, based on the exchange of Hopx, a homeobox protein reported as specific marker for $4+$ cells $^{[79]}$.

\subsection{Cell regeneration and plasticity}

Following crypt damage, the quiescent Paneth cell precursors can revert back to Lgr5+ stem cells for stem cell replacement and regeneration and thus serve as reserve stem cells. They demonstrated the plasticity of intestinal stem cells ${ }^{[73]}$. A new probe of the quiescent stem cells came from the induction of histone H2B labeled by yellow fluorescent protein (YFP) that labeled all crypt cells, except Paneth cells. After pulse, chase and dilution of cell division, labeled LRCs in the crypt base were identified by markers for entero-endocrine cells, (Lgr5+) CBC and +4 cells, including Paneth cells. The quiescent +4 cells were also genetically marked with histone $\mathrm{H} 2 \mathrm{~B}$ using a duel split Cre recombinase fusion, then followed in vivo. This marking separated the non-dividing Paneth cells from all the other cell types. After irradiation induced damage the same LRC cells became self-renewing, multipotent stem cells. The quiescent cells are progenitor-like, capable of reverting to the stem cell state $^{[79]}$. Further support was found in a study of the TA cells at the +5 position that were identified as secretory multipotent progenitor cells. These cells expressed the Notch ligand, Delta-like (Dll1) and by lineage tracing, they could generate clones of goblet cells, enteroendocrine cells, Paneth cells and tuft cells. When exposed to irradiation injury that destroyed the CBC stem cells, the DLL1+ cells became Lgr5+, CBC stem cells, another measure of adaptability ${ }^{[80,81]}$. These observations indicate the intestinal crypt contains several populations of reserve stem cells. The plasticity of these reserve progenitors is regulated by the stem cell niche that produces the necessary signals to direct the committed progenitors back into multipotent adult stem cells. In an acute injury, the Lgr5 cells are lost but the Paneth cells and +4 cells are intact. The surviving +4 cells reserve stem cells restore the Lgr5+ stem cell pool 
for epithelial cell renewal via a TA population that generates a population of Paneth precursor cells which ultimately differentiate into Paneth cells ${ }^{[81]}$. A model of crypt dynamics with in vivo experimentation was presented to analyze the dynamics of ISCs during homeostasis, regenerative repair and tumorigenesis ${ }^{[82]}$. They noted that active CBC stem cells and +4 quiescent stem cells express functional and molecular markers. Also, CBC stem cells and differentiated entero-endocrine cells high on the villus express Sox9 and EGFP, verifying their connection. Stem cells at the +4 position have been further identified by immuno-staining and lineage tracing (Bmi1, Lrigl, mTert and Hopx), which indicated they are long lived, slow cycling, can be activated by injury, and can repopulate the CBC pool. Also, two intestinal cell markers, Bmil and mTert (mouse teleomerase reverse transcriptase) have identified that there are functionally distinct populations. Lgr5 marks active ISCs that are regulated by Wnt, while Bmil marks quiescent ISCs that do not respond to Wnt regulation ${ }^{[83]}$

\section{Summary}

Adult somatic stem cells are tissue-specific quiescent or active selfrenewing reserve cells that exist in microenvironmental cellular niches. The activated stem cells are accompanied by precursor progenitor cells in various degrees of specialization for terminal differentiated cells. Components of the niche modulates the stem and precursor cell functions. This continuum of cells nominally serves to regenerate cells for tissue/organ homeostasis and exhibits considerable multipotency that decreases as differentiation progresses to the final specialized cell. This plasticity is often attributed to the active recruitment of particular progenitor cells for specific needs. Another view is that individual populations of the stem cell lineage can produce a functional plasticity through modulation of their differentiation by dedifferentiation or transdifferentiation ${ }^{[13]}$. Rather than a unique catalog of cells, it has been proposed that variations in the stem cell lineage might be achieved transiently ${ }^{[7]}$. Evidence indicates that the niche does not always elect predetermination of the stem cell lineage but individual signaling imposes specific behavior on them ${ }^{[67]}$. A second aspect is stem cell response to damage produced by trauma. One proposal is the presence of facultative stem cells, a subset of daughter cells that can revert to stem cells for damage control $^{[84]}$. It is possible that modulation of differentiation can occur in regeneration of both normal stem cell lineage and trauma induced events. The active selection of poised progenitor cells by the niche is likely the avenue for normal homeostasis, but temporary demands from physiological changes could trigger a rapid replenishment of the stem cell lineage through modulation of the differentiated state. This is illustrated in the niche imposed changes in the behavior of MaSCs during pregnancy and lactation that were not the result of predetermination $^{[67]}$. Other stem cells elicit a default response. For example, when HSC cells are depleted, newly introduced differentiated cells behave as the original stem cells ${ }^{[20]}$. When the neural stem cell pool is depleted, glial cells and melanocytes dedifferentiate into an intermediate bipotent progenitor ${ }^{[44]}$. After depletion of intestinal stem cells, the label retaining cells can dedifferentiate into Lgr5 intestinal cells ${ }^{[79]}$. In response to injury, differentiated Schwann cells can dedifferentiate into stem-like cells ${ }^{[45]}$. Similarly, after destruction of the stem cell population from trauma, the stem cells may be replaced by differentiated osteoblasts from the niche which would evoke their de-differentiation ${ }^{[22]}$. Following destruction of the crypt columnar cells, the Notch ligand expressing cells become Lgr5+ CBC stem cells ${ }^{[80,81]}$. After damage to the intestinal crypt, the Paneth cells can also revert to Lgr5 cells ${ }^{[72]}$. Evidence suggests that there are functionally different quiescent and active stem cells in the intestine. The Bmil quiescent cells respond to injury for regeneration, whereas the Lgr5 population acts during homeostasis ${ }^{[3]}$. The regulatory potential of the niche also affects differentiation of cells from stem cells of different cell lineages ${ }^{[68]}$. Neural stem cells are modified to those of the mammary gland after transplantation into the mammary crypt ${ }^{[85]}$. Similarly, the mammary niche can redirect the cell fate of spermatogenic cells ${ }^{[86]}$. Both examples illustrate the regulatory power of the niche to produce adaptive changes in differentiation of the stem cell lineage.

\section{References}

1. Slack JM. Origin of stem cells in organogenesis. Science. 2008;322(5907):1498-501.

2. Jones DL, Wagers AJ. No place like home: anatomy and function of the stem cell niche. Nat Rev Mol Cell Biol. 2008;9(1):11-21.

3. Spradling A, Drummond-Barbosa D, Kai T. Stem cells find their niche. Nature.2001;414(6859):98-104.

4. Sell S. Stem cell origin of cancer and differentiation therapy. Crit Rev Oncol Hematol. 2004 ;51(1):1-28.

5. Scadden DT. The stem-cell niche as an entity of action. Nature. 2006;441(7097):1075-9.

6. Moore KA, Lemischka IR. Stem cells and their niches. Science. 2006;311(5769):1880-5.

7. Tropepe V, Turksen K. The ontogeny of somatic stem cells. Stem Cell Rev. 2012;8(2):548-50.

8. Cheung TH, Rando TA. Molecular regulation of stem cell quiescence. Nat Rev Mol Cell Biol. 2013;14(6):329-40.

9. Clevers H, Loh KM, Nusse R. Stem cell signaling. An integral program for tissue renewal and regeneration: Wnt signaling and stem cell control. Science.2014;346(6205):1248012.

10. Tang DG. Understanding cancer stem cell heterogeneity and plasticity. Cell Res. 2012;22(3):457-72.

11. Li L, Clevers H. Coexistence of quiescent and active adult stem cells in mammals. Science. 2010;327(5965):542-5.

12. Takeda N, Jain R, LeBoeuf MR, Wang Q, Lu MM, Epstein JA. Interconversion between intestinal stem cell populations in distinct niches. Science. 2011;334(6061):1420-4.

13. Blau HM, Brazelton TR, Weimann JM. The evolving concept of a stem cell: entity or function? Cell. 2001;105(7):829-41.

14. Clevers H. STEM CELLS. What is an adult stem cell? Science. 2015;350(6266):1319-20.

15. Orkin SH, Zon LI. Hematopoiesis: an evolving paradigm for stem cell biology.Cell. 2008;132(4):631-44.

16. Phillips RL, Ernst RE, Brunk B, Ivanova N, Mahan MA, Deanehan JK, Moore KA, Overton GC, Lemischka IR. The genetic program of hematopoietic stem cells. Science. 2000;288(5471):1635-40.

17. Ding L, Morrison SJ. Haematopoietic stem cells and early lymphoid progenitors occupy distinct bone marrow niches. Nature. 2013;495(7440):231-5.

18. Yokota T, Oritani K, Butz S, Kokame K, Kincade PW, Miyata T, Vestweber D, Kanakura Y. The endothelial antigen ESAM marks primitive hematopoietic progenitors throughout life in mice. Blood. 2009;113(13):2914-23.

19. Morrison SJ, Spradling AC. Stem cells and niches: mechanisms that promote stem cell maintenance throughout life. Cell. 2008;132(4):598-611.

20. Seita J, Weissman IL. Hematopoietic stem cell: self-renewal versus differentiation. Wiley Interdiscip Rev Syst Biol Med. 2010;2(6):640-53.

21. Rieger MA, Hoppe PS, Smejkal BM, Eitelhuber AC, Schroeder T. Hematopoietic cytokines can instruct lineage choice. Science. 2009;325(5937):217-8.

22. Ding L, Saunders TL, Enikolopov G, Morrison SJ. Endothelial and perivascular cells maintain haematopoietic stem cells. Nature. 2012;481(7382):457-62.

23. Tweedell KS. The urodele limb regeneration blastema: the cell potential. ScientificWorldJournal. 2010;10:954-71.

24. Cabezas-Wallscheid N, Trumpp A. STEM CELLS. Potency finds its niches. Science. 2016;351(6269):126-7.

25. Khan JA, Mendelson A, Kunisaki Y, Birbrair A, Kou Y, ArnalEstapé A, Pinho S, Ciero P, Nakahara F, Ma'ayan A, Bergman A, Merad M, Frenette PS. Fetal liver hematopoietic stem cell niches associate with portal vessels. Science. 2016;351(6269):176-80. 
26. Notta F, Zandi S, Takayama N, Dobson S, Gan OI, Wilson G, Kaufmann KB, McLeod J, Laurenti E, Dunant CF, McPherson JD, Stein LD, Dror Y, Dick JE. Distinct routes of lineage development reshape the human blood hierarchy across ontogeny. Science. 2016 Jan 8;351(6269):aab2116.

27. Paul F, Arkin Y, Giladi A, Jaitin DA, Kenigsberg E, Keren-Shaul H, Winter D, Lara-Astiaso D, Gury M, Weiner A, David E, Cohen N, Lauridsen FK, Haas S, Schlitzer A, Mildner A, Ginhoux F, Jung S, Trumpp A, Porse BT, Tanay A, Amit I. Transcriptional Heterogeneity and Lineage Commitment in Myeloid Progenitors. Cell. $2015 ; 163(7): 1663-77$.

28. Gage FH. Mammalian neural stem cells. Science. 2000;287(5457):1433-8.

29. Doetsch F, Caillé I, Lim DA, García-Verdugo JM, Alvarez-Buylla A.Subventricular zone astrocytes are neural stem cells in the adult mammalian brain. Cell. 1999;97(6):703-16.

30. Götz M, Huttner WB. The cell biology of neurogenesis. Nat Rev Mol Cell Biol. 2005;6(10):777-88.

31. Anthony TE, Klein C, Fishell G, Heintz N. Radial glia serve as neuronal progenitors in all regions of the central nervous system. Neuron. 2004;41(6):881-90.

32. Alvarez-Buylla A, García-Verdugo JM, Tramontin AD. A unified hypothesis on the lineage of neural stem cells. Nat Rev Neurosci. 2001;2(4):287-93.

33. Ihrie RA, Alvarez-Buylla A. Cells in the astroglial lineage are neural stem cells. Cell Tissue Res. 2008;331(1):179-91

34. Kriegstein A, Alvarez-Buylla A. The glial nature of embryonic and adult neural stem cells. Annu Rev Neurosci. 2009;32:149-84.

35. Grabel L. Developmental origin of neural stem cells: the glial cell that could. Stem Cell Rev. 2012;8(2):577-85.

36. Lin R, Iacovitti L. Classic and novel stem cell niches in brain homeostasis and repair. Brain Res. 2015;1628(Pt B):327-42.

37. Ottone C, Krusche B, Whitby A, Clements M, Quadrato G, Pitulescu ME, Adams RH, Parrinello S. Direct cell-cell contact with the vascular niche maintains quiescent neural stem cells. Nat Cell Biol. 2014;16(11):1045-56.

38. Kawaguchi D, Furutachi S, Kawai H, Hozumi K, Gotoh Y. Dll1 maintains quiescence of adult neural stem cells and segregates asymmetrically during mitosis. Nat Commun. 2013;4:1880.

39. 39. Jones KM, Sarić N, Russell JP, Andoniadou CL, Scambler PJ, Basson MA. CHD7 maintains neural stem cell quiescence and prevents premature stem cell depletion in the adult hippocampus. Stem Cells. 2015;33(1):196-210.

40. Silva-Vargas V, Crouch EE, Doetsch F. Adult neural stem cells and their niche: a dynamic duo during homeostasis, regeneration, and aging. Curr Opin Neurobiol. 2013;23(6):935-42.

41. Riquelme PA, Drapeau E, Doetsch F. Brain micro-ecologies: neural stem cell niches in the adult mammalian brain. Philos Trans R Soc Lond B Biol Sci. 2008 ;363(1489):123-37.

42. Fuentealba LC, Obernier K, Alvarez-Buylla A. Adult neural stem cells bridge their niche. Cell Stem Cell. 2012;10(6):698-708.

43. Tong CK, Chen J, Cebrián-Silla A, Mirzadeh Z, Obernier K, Guinto CD, Tecott LH, García-Verdugo JM, Kriegstein A, AlvarezBuylla A. Axonal control of the adult neural stem cell niche. Cell Stem Cell. 2014;14(4):500-11.

44. Dupin E, Real C, Glavieux-Pardanaud C, Vaigot P, Le Douarin NM. Reversal of developmental restrictions in neural crest lineages: transition from Schwann cells to glial-melanocytic precursors in vitro. Proc Natl Acad Sci U S A. 2003;100(9):5229-33.

45. Jessen KR, Mirsky R. The origin and development of glial cells in peripheral nerves. Nat Rev Neurosci. 2005;6(9):671-82.

46. Takahashi Y, Sipp D, Enomoto H. Tissue interactions in neural crest cell development and disease. Science. 2013;341(6148):860-3.

47. Masaki T, Qu J, Cholewa-Waclaw J, Burr K, Raaum R, Rambukkana A. Reprogramming adult Schwann cells to stem celllike cells by leprosy bacilli promotes dissemination of infection. Cell. 2013;152(1-2):51-67.

48. Xin L, Ide H, Kim Y, Dubey P, Witte ON. In vivo regeneration of murine prostate from dissociated cellpopulations of postnatal epithelia and urogenital sinus mesenchyme . Proc Natl Acad Sci U S A. 2003; 100: (Suppl 1) 11896-903.
49. Xin L, Lukacs RU, Lawson DA, Cheng D, Witte ON. Selfrenewal and multilineage differentiation in vitro from murine prostate stem cells. Stem Cells. 2007;25(11):2760-9.

50. Burger PE, Xiong X, Coetzee S, Salm SN, Moscatelli D, Goto K, Wilson EL. Sca-1 expression identifies stem cells in the proximal region of prostatic ducts with high capacity to reconstitute prostatic tissue. Proc Natl Acad Sci U S A. 2005;102(20):7180-5.

51. Leong KG, Wang BE, Johnson L, Gao WQ. Generation of a prostate from a single adult stem cell. Nature. 2008;456(7223):804-8.

52. Lawson DA, Xin L, Lukacs RU, Cheng D, Witte ON. Isolation and functional characterization of murine prostate stem cells. Proc Natl Acad Sci U S A. 2007 ;104(1):181-6.

53. Richardson GD, Robson CN, Lang SH, Neal DE, Maitland NJ, Collins AT. CD133, a novel marker for human prostatic epithelial stem cells. J Cell Sci. 2004 ;117(Pt 16):3539-45.

54. Garraway IP, Sun W, Tran CP, Perner S, Zhang B, Goldstein AS, Hahm SA, Haider M, Head CS, Reiter RE, Rubin MA, Witte ON. Human prostate sphere-forming cells represent a subset of basal epithelial cells capable of glandular regeneration in vivo. Prostate. 2010;70(5):491-501.

55. Goldstein AS, Stoyanova T, Witte ON. Primitive origins of prostate cancer: in vivo evidence for prostate-regenerating cells and prostate cancer-initiating cells. Mol Oncol. 2010;4(5):385-96.

56. Ousset M, Van Keymeulen A, Bouvencourt G, Sharma N, Achouri Y, Simons BD, Blanpain C. Multipotent and unipotent progenitors contribute to prostate postnatal development. Nat Cell Biol. 2012;14(11):1131-8.

57. Shahi P, Seethammagari MR, Valdez JM, Xin L, Spencer DM. Wnt and Notch pathways have interrelated opposing roles on prostate progenitor cell proliferation and differentiation. Stem Cells. 2011;29(4):678-88

58. Luo W, Rodriguez M, Valdez JM, Zhu X, Tan K, Li D, Siwko S, Xin L, Liu M. Lgr4 is a key regulator of prostate development and prostate stem cell differentiation. Stem Cells. 2013;31(11):2492505 .

59. Strand DW, Goldstein AS. The many ways to make a luminal cell and a prostate cancer cell. Endocr Relat Cancer. 2015;22(6):T18797.

60. Faraldo MM, Taddei-De La Hosseraye I, Teulière J, Deugnier MA, Moumen M, Thiery JP, Glukhova MA. [Mammary gland development: Role of basal myoepithelial cells]. J Soc Biol. 2006;200(2):193-8.

61. Petersen OW, Polyak K. Stem cells in the human breast. Cold Spring Harb Perspect Biol. 2010;2(5):a003160.

62. Shackleton M, Vaillant F, Simpson KJ, Stingl J, Smyth GK, Asselin-Labat ML, Wu L, Lindeman GJ, Visvader JE. Generation of a functional mammary gland from a single stem cell. Nature. 2006;439(7072):84-8

63. Wang D, Cai C, Dong X, Yu QC, Zhang XO, Yang L, Zeng YA. Identification of multipotent mammary stem cells by protein $\mathrm{C}$ receptor expression. Nature. $2015 ; 517(7532): 81-4$.

64. Van Keymeulen A, Rocha AS, Ousset M, Beck B, Bouvencourt G, Rock J, Sharma N, Dekoninck S, Blanpain C. Distinct stem cells contribute to mammary gland development and maintenance. Nature. 2011;479(7372):189-93.

65. Hassiotou F, Beltran A, Chetwynd E, Stuebe AM, Twigger AJ, Metzger P, Trengove N, Lai CT, Filgueira L, Blancafort P, Hartmann PE. Breastmilk is a novel source of stem cells with multilineage differentiation potential. Stem Cells. 2012;30(10):2164-74

66. Rios AC, Fu NY, Lindeman GJ, Visvader JE. In situ identification of bipotent stem cells in the mammary gland. Nature. 2014;506(7488):322-7.

67. LaBarge MA, Nelson CM, Villadsen R, Fridriksdottir A, Ruth JR, Stampfer MR, Petersen OW, Bissell MJ. Human mammary progenitor cell fate decisions are products of interactions with combinatorial microenvironments. Integr Biol (Camb). 2009;1(1):70-9.

68. LaBarge MA. On stem cells in the human breast. Cold Spring Harb Perspect Biol. 2012;4(5). pii: a013441. 
69. Mishra L, Shetty K, Tang Y, Stuart A, Byers SW. The role of TGFbeta and Wnt signaling in gastrointestinal stem cells and cancer. Oncogene. 2005;24(37):5775-89.

70. Medema JP, Vermeulen L. Microenvironmental regulation of stem cells in intestinal homeostasis and cancer. Nature. 2011;474(7351):318-26.

71. Cheng H, Leblond CP. Origin, differentiation and renewal of the four main epithelial cell types in the mouse small intestine. V. Unitarian Theory of the origin of the four epithelial cell types. Am J Anat. 1974;141(4):537-61.

72. Clevers H. The intestinal crypt, a prototype stem cell compartment. Cell. 2013;154(2):274-84.

73. Clevers H, Batlle E. SnapShot: the intestinal crypt. Cell. 2013;152(5):1198-1198.e2.

74. Clevers H. Stem Cells: A unifying theory for the crypt. Nature. 2013;495(7439):53-4.

75. Barker N, van de Wetering M, Clevers H. The intestinal stem cell. Genes Dev. 2008;22(14):1856-64.

76. Potten CS. Extreme sensitivity of some intestinal crypt cells to X and gamma irradiation. Nature. 1977;269(5628):518-21.

77. Li VS, Clevers H. In vitro expansion and transplantation of intestinal crypt stem cells. Gastroenterology. 2012;143(1):30-4

78. Sangiorgi E, Capecchi MR. Bmil is expressed in vivo in intestinal stem cells. Nat Genet. 2008;40(7):915-20.

79. Buczacki SJ, Zecchini HI, Nicholson AM, Russell R, Vermeulen L, Kemp R, Winton DJ. Intestinal label-retaining cells are secretory precursors expressing Lgr5. Nature. 2013;495(7439):65-9.

80. van Es JH, Sato T, van de Wetering M, Lyubimova A, Nee AN, Gregorieff A, Sasaki N, Zeinstra L, van den Born M, Korving J, Martens AC, Barker N, van Oudenaarden A, Clevers H. D111+ secretory progenitor cells revert to stem cells upon crypt damage. Nat Cell Biol. 2012;14(10):1099-104.

81. Barker N. Adult intestinal stem cells: critical drivers of epithelial homeostasis and regeneration. Nat Rev Mol Cell Biol. 2014;15(1):19-33.

82. Carulli AJ, Samuelson LC, Schnell S. Unraveling intestinal stem cell behavior with models of crypt dynamics. Integr Biol (Camb). 2014;6(3):243-57.

83. Yan KS, Chia LA, Li X, Ootani A, Su J, Lee JY, Su N, Luo Y, Heilshorn SC, Amieva MR, Sangiorgi E, Capecchi MR, Kuo CJ. The intestinal stem cell markers Bmi1 and Lgr5 identify two functionally distinct populations. Proc Natl Acad Sci U S A. 2012;109(2):466-71

84. Visvader JE, Clevers H. Tissue-specific designs of stem cell hierarchies. Nat Cell Biol. 2016;18(4):349-55.

85. Booth BW, Mack DL, Androutsellis-Theotokis A, McKay RD, Boulanger CA, Smith GH. The mammary microenvironment alters the differentiation repertoire of neural stem cells. Proc Natl Acad Sci U S A. 2008;105(39):14891-6.

86. Boulanger CA, Mack DL, Booth BW, Smith GH. Interaction with the mammary microenvironment redirects spermatogenic cell fate in vivo. Proc Natl Acad Sci U S A. 2007;104(10):3871-6.

$\begin{array}{ll}\text { Abbreviation } \\ \text { Ang1 } & \text { Angiopoietin, vascular growth factor } \\ \text { Bmi1 } & \text { Proto-oncogene } \\ \text { BMP } & \text { Bone morphogenetic protein } \\ \text { c-kit } & \text { Receptor for tyrosine kinase } \\ \text { Cre- } & \text { Recombinase site specific enzyme for DNA } \\ \text { ECM } & \text { Extracellular matrix } \\ \text { EFFR } & \text { Epidermal growth factor receptor } \\ \text { FACS } & \text { Fluorescence activated cell sorting } \\ \text { FGF } & \text { Fibroblast growth factor } \\ \text { GFAP } & \text { Glial fibrillary acidic protein } \\ \text { Hox } & \text { Transmission factors for axial patterning } \\ \text { Hopx } & \text { Homeobox for lethality } \\ \text { IGF } & \text { Insulin-like growth factor } \\ \text { LaxZ } & \text { Transgene for } \beta \text { galactosidase } \\ \text { Lrg5 } & \text { Leucine g protein receptor } \\ \text { Lin- } & \text { Lineage (negative) antigen } \\ \text { MEP } & \text { Myoepithelial cell } \\ \text { Notch } & \text { Transmembrane protein signaling pathway } \\ \text { OPN } & \text { Osteopontin, bone matrix protein } \\ \text { Pax6 } & \text { Paired box transcription factor, brain } \\ \text { PDGF } & \text { Platlet derived growth factor } \\ \text { Pten } & \text { Phosphatase and tensin protein } \\ \text { SHH } & \text { Sonic hedgehog signaling pathway } \\ \text { SCA-1 } & \text { General stem cell antigen } \\ \text { Sox2 } & \text { Sex determining transcription factor } \\ \text { TGAF } \beta & \text { Transforming growth factor signaling pathway } \\ \text { Tie } & \text { Angiopoietin receptor } \\ \text { Trop2 } & \text { Trophoblast cell surface glycoprotein } \\ \text { VEGF } & \text { Vascular endothelial growth factor } \\ \text { Wnt } & \text { Signaling transduction pathway } \\ & \end{array}$


Stem cell adaptability

\section{Potential Conflicts of Interests}

None

Corresponding Author

Kenyon S. Tweedell, Department of Biological Sciences, University of Notre Dame, Notre Dame IN 46556 USA;

Email Tweedell.1@ nd.edu 